\section{BMJ Open Respiratory Research}

\title{
Step oximetry test: a validation study
}

\author{
Benjamin Daniel Fox, ${ }^{1,2,3}$ Nadav Sheffy, ${ }^{1}$ Baruch Vainshelboim, ${ }^{1}$ Leonardo Fuks, ${ }^{1}$ \\ Mordechai R Kramer ${ }^{1,3}$
}

To cite: Fox BD, Sheffy N, Vainshelboim B, et al. Step oximetry test: a validation study. BMJ Open Resp Res 2018;5:e000320. doi:10.1136/ bmjresp-2018-000320

Received 31 May 2018 Revised 4 July 2018 Accepted 5 July 2018
Check for updates

(C) Author(s) (or their employer(s)) 2018. Re-use permitted under CC BY-NC. No commercial re-use. See rights and permissions. Published by BMJ.

${ }^{1}$ Pulmonary Institute, Rabin Medical Center, Petah Tikva, Israel

${ }^{2}$ Pulmonary Institute, Assaf Harofeh Medical Center, Zerifin, Israel

${ }^{3}$ Faculty of Medicine, Tel Aviv University, Tel Aviv, Israel

Correspondence to Dr Benjamin Daniel Fox; benfox@post.tau.ac.il

\section{ABSTRACT}

Introduction Step climbing is a potentially useful modality for testing exercise capacity. However, there are significant variations between test protocols and lack of consistent validation against gold standard cycle ergometry cardiopulmonary exercise testing (CPET). The purpose of the study was to validate a novel technique of exercise testing using a dedicated device.

Methods We built a step oximetry device from an adapted aerobics step and pulse oximeter connected to a computer. Subjects performed lung function tests, a standard incremental cycle CPET and also a CPET while stepping on and off the step oximetry device to maximal exertion. Data from the step oximetry device were processed and correlated with standard measurements of pulmonary function and cycle CPET.

Results We recruited 89 subjects (57 years, 50 men). Oxygen uptake $\left(\mathrm{VO}_{2}\right)$ was $0.9 \mathrm{~mL} / \mathrm{kg} / \mathrm{min}(95 \% \mathrm{Cl}-3.6$ to 5.4) higher in the step test compared with the gold standard cycle CPET, $p<0.001 . \mathrm{VO}_{2}$ in the two techniques was highly correlated $(R=0.87, p<0.001)$. Work rate during stair climbing showed the best correlation with $\mathrm{VO}_{2}(\mathrm{R}=0.69, \mathrm{p}<0.0001)$. Desaturation during step climbing correlated negatively with diffusion capacity for carbon monoxide $(r=-0.43, p<0.005)$. No adverse events occurred.

Conclusions The step oximetry test was a maximal test of exertion in the subjects studied, achieving slightly higher $\mathrm{VO}_{2}$ than during the standard test. The test was safe to perform and well tolerated by the patients. Parameters derived from the step oximetry device correlated well with gold standard measurements. The step oximetry test could become a useful and standardisable exercise test for clinical settings where advanced testing is not available or appropriate.

\section{INTRODUCTION}

Difficulty in climbing stairs is a frequent complaint in patients with exercise intolerance from any cause. It is also a familiar and necessary form of exercise for all patients, as opposed to treadmill walking or pedalling on a stationary exercise bicycle. In spite of this, there are only a small number of studies evaluating the physiology of stair climbing. These studies vary in terms of testing protocols, step height, total height climbed (usually limited by the size of the hospital building), the factor determining end of test (height, time) and the endpoint evaluated (height gained, climbing time, velocity, power). Step testing is a reproducible test across different protocols. ${ }^{1-5}$ In general, step climbing performance correlates well with the gold standard peak oxygen uptake $\left(\mathrm{VO}_{2}\right.$ peak $)$, both in height-limited and time-limited tests, although in most cases $\mathrm{VO}_{2}$ peak was not measured during the step climbing itself. ${ }^{2-11}$ In two recent studies, $\mathrm{VO}_{2}$ was measured directly during step climbing and cycle ergometry, with conflicting results regarding the relationship between $\mathrm{VO}_{2}$ with the different tests. ${ }^{112}$

The aim of this study was to measure $\mathrm{VO}_{2}$ during a standardised, laboratory-based step climbing test using a dedicated device and compare performance with the gold standard test of cycle ergometry. In addition, we explored several novel parameters derived from the technique.

\section{METHODS}

The study protocol was approved by the Rabin Medical Center Ethics Committee. All subjects gave written informed consent.

\section{Subject selection}

Subjects were invited to participate in the study if they had been referred for cycle ergometry cardiopulmonary exercise testing (CPET) by their physician. Patients with significant neurological or musculoskeletal limitation which could affect their step climbing ability were excluded.

\section{Patient testing and data collection}

All patients attending for CPET performed spirometry and single breath diffusion capacity for carbon monoxide (DLCO) as a part of their workup, according to standard guidelines (ZAN-500 system, nSpire Health, Longmont, CO). ${ }^{13} 14$ The cycle ergometry CPET was performed in accordance with established guidelines (CardiO2Max, Medical Graphics, St Paul, MN). ${ }^{15}$ In brief, patients rested on the cycle ergometer for $2 \mathrm{~min}$, then $2 \mathrm{~min}$ of unloaded cycling at $60 \mathrm{rpm}$ followed by a $15 \mathrm{~W} / \mathrm{min}$ ramp. All parameters measured 


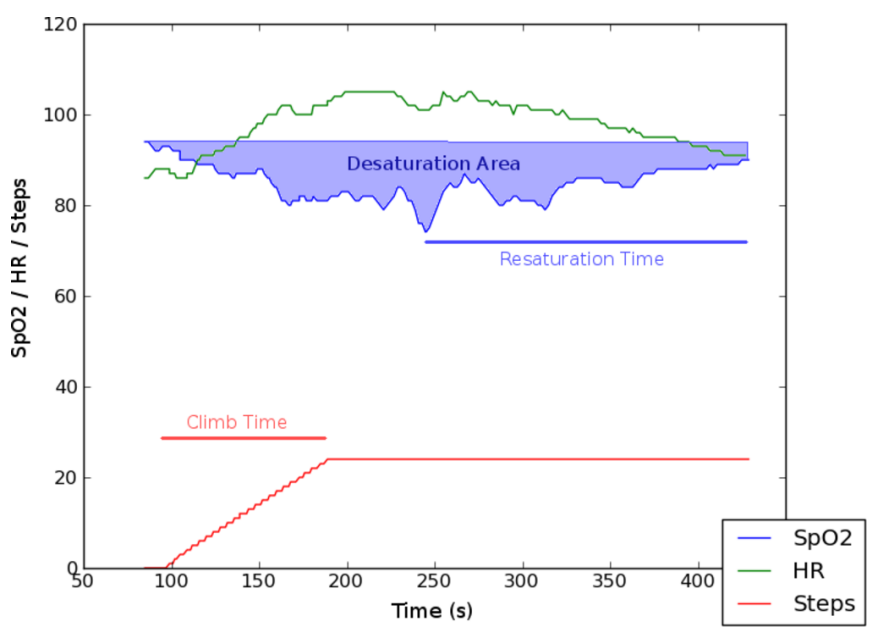

Figure 1 Representative plot from step oximetry device, annotated to demonstrate some of the step oximetry parameters.

during CPET were averaged over $30 \mathrm{~s}$. End of test was defined by the earliest occurrence of patient exhaustion or failure to maintain $60 \mathrm{rpm}$ pedalling, achievement of $\mathrm{VO}_{2}$ peak predicted, respiratory exchange ratio $>1.15$ or heart rate (HR) $>90 \%$ predicted. ${ }^{15}$ Anaerobic threshold was determined with the V-slope technique. ${ }^{15}$ Following the test, patients rested on a chair for at least $90 \mathrm{~min}$ before doing the step oximetry test, as detailed below.

\section{Step oximetry test: patient monitoring/data collection}

Step testing was performed while the subject was monitored with the metabolic cart of the cycle ergometry CPET device. The step oximetry device was built by the investigators from a $17 \mathrm{~cm}$ high 'aerobics' step, adapted with pressure sensors that transmit a signal to the computer when the subject mounts the step with both feet. A pulse oximeter (Radical 7, Massimo, Irvine, CA) outputted HR and $\mathrm{SpO}_{2}$ data every $6 \mathrm{~s}$ to the computer. Integration of time, step count, $\mathrm{HR}$ and $\mathrm{SpO}_{2}$ were performed by a computer program. Before the step test, the subject read a standard written instruction, stating that they should climb for as long as possible at a constant rate (20 per min) until their self-perceived limit was reached. A brief demonstration of stepping was performed by the study physician. During the step test the subject mounted and dismounted from the step with one foot after the other until he/she reached their self-perceived maximal exertion or an arbitrary limit of 200 steps (34 m vertical climb). Other than a verbal confirmation that the subject was climbing correctly, the test was performed unencouraged. Subjects were not aware of the 200 steps limit and were not allowed to view the computer monitor during the test. ${ }^{4}$ At the end of the step test the subject rested on a chair while monitoring of $\mathrm{HR} / \mathrm{SpO}_{2}$ continued until recovery (HR within $5 \%$ of baseline and $\mathrm{SpO}_{2}$ with $2 \%$ of baseline value).

Postprocessing of the data was performed by a computer algorithm developed by the investigators, based on equations from classical Newtonian physics.
A graphical representation of a typical test is shown in figure 1. Exercise performance indices were: total height gained; climbing time; mean vertical climbing velocity; total work against gravity (energy: mass*total height gained* $g$ where mass was body weight in $\mathrm{kg}$ ); and work rate (energy divided by climbing time). We also derived the 'Climb Index' from velocity and height gained. From the HR data we calculated the per cent use of predicted heart rate reserve (220-age) and the slope of HR recovery regression line. Desaturation indices were defined as: maximum absolute drop in $\mathrm{SpO}_{2}$ from baseline value (desaturation); desaturation area (the integration of time with $\mathrm{SpO}_{2}$ values below 95\%); and the mean deviation of $\mathrm{SpO}_{2}$ below 95\% (saturation deviation). In patients with $>2 \%$ absolute desaturation we calculated the time to recovery of $\mathrm{SpO}_{2}$ to within $2 \%$ of baseline values (resaturation time).

\section{Data analysis}

The primary outcome of interest was the agreement between $\mathrm{VO}_{2}$ on both tests, assessed by the Bland-Altman technique. ${ }^{16^{2}}$ Secondary endpoints were the correlations between $\mathrm{VO}_{2}$ and the derived exercise performance indices generated by the step oximetry algorithm, or DLCO and the desaturation indices. Categorical data were summarised and analysed using the $\mathrm{X}^{2}$ test. Continuous data were summarised as mean (95\% CIs) or median (IQR) as appropriate. Parameters with significant right skew were $\log$ transformed and analysed and mean, 95\% CI reported in their original units after an anti-log transformation. ${ }^{17}$ The correlation between parameters of interest was by Pearson's R or Spearman's rho as appropriate. Given the large number of possible comparisons, we applied the Holm-Bonferroni correction and reported

\begin{tabular}{|c|c|}
\hline Age (years) & 57.7 (54.3 to 60.2$)$ \\
\hline Sex (M:F) & $50: 39$ \\
\hline Weight (kg) & 74.4 (70.7 to 78.2$)$ \\
\hline Body mass index (kg/m) & 27.5 (26.5 to 29.1$)$ \\
\hline \multirow[t]{6}{*}{ Principal diagnosis } & Lung transplant recipient, 28 \\
\hline & $\begin{array}{l}\text { Chronic obstructive pulmonary } \\
\text { disease, } 17\end{array}$ \\
\hline & $\begin{array}{l}\text { Pulmonary arterial } \\
\text { hypertension, } 10\end{array}$ \\
\hline & Cardiac failure, 7 \\
\hline & Interstitial lung disease, 5 \\
\hline & Others, 22 \\
\hline FVC (\% predicted) & 68 (53 to 65) \\
\hline $\mathrm{FEV}_{1}$ (\% predicted) & 59 (53 to 64) \\
\hline DLCO (\% predicted) & 48 (42 to 54$)$ \\
\hline
\end{tabular}

DLCO, diffusion capacity for carbon monoxide; $\mathrm{FEV}_{1}$, forced expiratory volume in $1 \mathrm{~s}$; FVC, forced vital capacity. 
Table 2 Comparison of CPET results with two techniques, the lower section of the table presents data available only from the step test

\begin{tabular}{|c|c|c|c|}
\hline & Cycle CPET & Step test CPET & MD (step cycle) \\
\hline Exercise time (s) & 307 (285 to 329) & 114 (98 to 130$)$ & $-194(-380 \text { to }-9)^{\star \star \star *}$ \\
\hline Work rate $(\mathrm{W})$ & 63 (58 to 69) & 38 (35 to 41$)$ & NA \\
\hline $\mathrm{VO}_{2}(\mathrm{~mL} / \mathrm{kg} / \mathrm{min})$ & $11.9(11.2$ to 12.7$)$ & $12.8(11.9$ to 13.7$)$ & $0.9(-3.6 \text { to } 5.4)^{\star \star *}$ \\
\hline $\mathrm{VO}_{2}(\%$ predicted $)$ & 54 (50 to 57$)$ & 58 (54 to 62) & $5(-17 \text { to } 26)^{\star \star \star}$ \\
\hline Heart rate $(\mathrm{bpm})$ & 115 (111 to 120$)$ & 114 (109 to 118$)$ & $-2(-32$ to 28$)$ \\
\hline Cardiac reserve (\%) & 71 (69 to 74$)$ & 68 (64 to 72) & $4(-35 \text { to } 28)^{\star}$ \\
\hline $\mathrm{O}_{2}$ pulse (mL/beat) & 7.5 (7.0 to 8.0$)$ & 8.1 (7.5 to 8.7$)$ & $0.7(-2.7 \text { to } 4.1)^{\star \star \star}$ \\
\hline VE (L/min) & 35.2 (32.7 to 37.8$)$ & 32.8 (30.3 to 35.5$)$ & $-2.2(-16.4 \text { to } 11.9)^{\star \star}$ \\
\hline Breathing reserve (\%) & 63 (60 to 67$)$ & 60 (56 to 64$)$ & $-3(-25 \text { to } 19)^{\star \star}$ \\
\hline RER & 1.07 (1.04 to 1.09$)$ & $0.86(0.84$ to 0.88$)$ & $-0.21(-0.45 \text { to } 0.03)^{\star * \star \star}$ \\
\hline Absolute desaturation & $3(2$ to 4$)$ & $5(4$ to 7$)$ & $-2(4 \text { to }-9)^{\star \star \star \star}$ \\
\hline Steps/min & & 19 (18 to 20$)$ & \\
\hline Total energy (kJ) & & 3.35 (2.84 to 3.95 ) & \\
\hline Climb index & & 2.77 (2.60 to 2.95 ) & \\
\hline Height gained (m) & & 4.72 (4.04 to 5.52$)$ & \\
\hline Velocity (m/min) & & 3.19 (3.01 to 3.35$)$ & \\
\hline Saturation deviation & & 0.6 (0.0 to 2.4$)$ & \\
\hline Desaturation area & & 48 (0 to 356$)$ & \\
\hline Resaturation time (s) & & 39 (13.5 to 70.0$)$ & \\
\hline
\end{tabular}

Data presented as mean $(95 \% \mathrm{Cl})$ or median (IQR). Mean difference (MD) between the groups is calculated by the Bland-Altman technique. Differences in work rate are not compared since the step oximetry system calculates only ascending work.

Significant difference between results in paired t-test:

${ }^{*} \mathrm{P}<0.05 ;{ }^{* *} \mathrm{P}<0.01 ;{ }^{* * *} \mathrm{P}<0.001 ;{ }^{* * *} \mathrm{P}<0.0001$.

CPET, cardiopulmonary exercise testing; NA, not applicable; RER, respiratory exchange ratio; VE, minute ventilation; $\mathrm{VO}_{2}$, peak oxygen uptake.

the uncorrected $\mathrm{p}$ values that remained significant after correction. ${ }^{18}$ All tests were two tailed and the predetermined level of significance was $\mathrm{p}<0.05$.

\section{RESULTS}

Eighty-nine patients were recruited (table 1). In the step test, mean height gained was $4.72 \mathrm{~m}(95 \% \mathrm{CI} 4.04$ to 5.52 ), equivalent to 28 steps. No patients reached the maximal limit of 200 steps (34 m ascent). Vertical velocity was $3.18 \mathrm{~m} / \mathrm{s}$ (95\% CI 3.01 to 3.35$)$. No adverse events occurred during the study.

Primary endpoint: agreement between $\mathrm{VO}_{2}$ on step oximetry and cycle ergometry

Oxygen uptake was $0.9 \mathrm{~mL} / \mathrm{kg} / \mathrm{min}$ (95\% CI -3.6 to 5.4) higher on the step oximetry test compared with the cycle CPET (table 2, figure 2). These higher levels of $\mathrm{VO}_{2}$ were achieved at a significantly shorter exercise time and lower use of cardiac/respiratory reserves than on the cycle CPET (table 2). $\mathrm{VO}_{2}$ in the two techniques was highly correlated, $\mathrm{R}=0.87 ; 95 \% \mathrm{CI}$ (0.81 to 0.92 ), $\mathrm{p}<0.001$. Of note it was difficult to establish the $\mathrm{VO}_{2}$ at anaerobic threshold during the step climbing test and so this comparison was excluded from the analysis.
Secondary endpoint: correlations between step oximetry parameters and $\mathrm{VO}_{2}$ and $\mathrm{DLCO}$

In all comparisons, step oximetry exercise performance indices correlated closely with $\mathrm{VO}_{2}$ achieved on both cycle ergometry and step oximetry, with good CIs (table 3). The closest correlation was observed for the work rate (W) calculated by the step oximetry algorithm $(\mathrm{R}=0.69)$ (figure 3). After applying the Bonferroni-Holms correction,

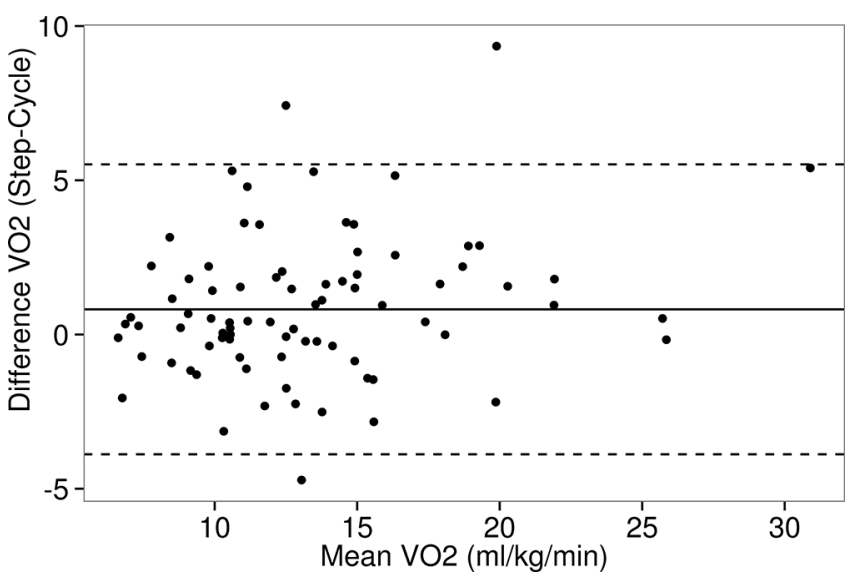

Figure 2 Bland-Altman plot of oxygen uptake $\left(\mathrm{VO}_{2}\right)$ during cycle ergometry and step oximetry. HR, heart rate. 
Table 3 Correlation table for step oximetry exercise performance indices-Pearson's R (95\% Cls)

\begin{tabular}{lll}
\hline Parameter & $\mathbf{V O}_{2}$ (cycle) & VO $_{2}$ (step) \\
\hline Work rate $(\mathrm{W})$ & $0.69(0.57$ to 0.78$)$ & $0.69(0.56$ to 0.78$)$ \\
\hline Total energy $(\mathrm{kJ})$ & $0.65(0.51$ to 0.75$)$ & $0.67(0.54$ to 0.77$)$ \\
Climb index & $0.57(0.42$ to 0.70$)$ & $0.60(0.45$ to 0.72$)$ \\
Height gained $(\mathrm{m})$ & $0.54(0.37$ to 0.67$)$ & $0.57(0.40$ to 0.70$)$ \\
Velocity $(\mathrm{m} / \mathrm{min})$ & $0.47(0.29$ to 0.62$)$ & $0.49(0.31$ to 0.63$)$ \\
Climb time $(\mathrm{s})$ & $0.41(0.22$ to 0.57$)$ & $0.41(0.22$ to 0.57$)$ \\
\hline
\end{tabular}

Parameters listed in descending order of R. All correlations reached statistical significance $(p<0.0001)$ after Bonferroni-Holms correction.

all correlations remained statistically significant. Pearson's $\mathrm{R}$ was similar between the two tests for each parameter.

We also correlated DLCO with the step oximetry desaturation indices (table 4). Saturation deviation, desaturation area and absolute desaturation all showed a significant negative linear correlation with DLCO, after applying the Bonferroni-Holms correction. Of note, absolute desaturation during cycle CPET also correlated negatively with DLCO.

\section{DISCUSSION}

We report the validation of a computerised laboratory-based step oximetry test on a dedicated device, compared with the gold standard cycle ergometry. We showed that $\mathrm{VO}_{2}$ peak on the step test typically exceeds that on the cycle ergometer, and that novel step oximetry exercise performance indices correlate well with $\mathrm{VO}_{2}$ peak.

Our step climbing protocol is a potentially attractive form of exercise testing for several reasons. The test appears to be a maximal exercise challenge for most patients, as demonstrated by the higher $\mathrm{VO}_{2}$ compared with the gold standard cycle CPET with similar increases in HR and ventilation. Furthermore, no patients in this cohort reached 200 steps in this study, and only $19 \%$ in a previous study. ${ }^{4}$ The

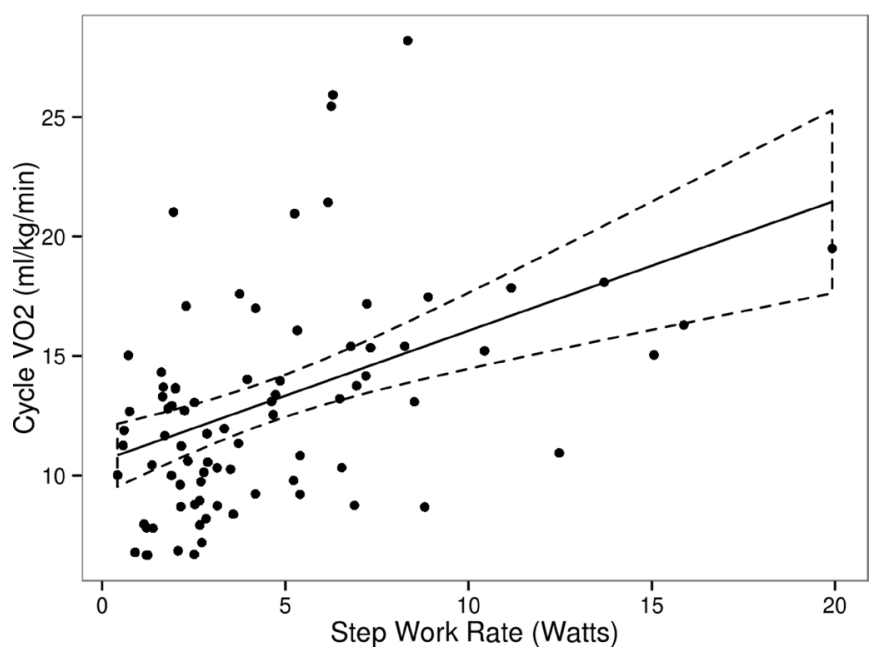

Figure 3 Correlation plot between work rate on the step climber and $\mathrm{VO}_{2}$ on the cycle ergometer.
Table 4 Correlation table for step oximetry oxygenation indices-Spearman's $r$ test

\begin{tabular}{ll}
\hline Parameter & DLCO \\
\hline Saturation deviation & $-0.43^{*}$ \\
\hline Absolute desaturation & $-0.42^{*}$ \\
\hline Desaturation area & $-0.40^{*}$ \\
Resaturation time & -0.14 \\
Cycle absolute desaturation & $-0.47^{*}$ \\
\hline
\end{tabular}

Parameters listed in descending order of $r$.

*Indicates statistical significance $(p<0.005)$ after Bonferroni-Holms correction.

DLCO, diffusion capacity for carbon monoxide.

protocol has previously been validated against WHO functional class and the $6 \mathrm{~min}$ walk (6MW) test. ${ }^{4}$ Our results are consistent with those of Dal Corso et al, who demonstrated slightly higher $\mathrm{VO}_{2}$ during step climbing among patients with chronic obstructive pulmonary disease compared with walking. ${ }^{1}$ Hansen et al studied healthy subjects and found that $\mathrm{VO}_{2}$ was $85 \%$ of that obtained during cycle ergometry. ${ }^{12}$ However, the healthy subjects in Hansen's group completed the test at submaximal HRs, strongly suggesting that step climbing may have not represented maximal exertion over the time limit or step limit used in the step test. In healthy controls doing the step oximetry test, we have shown a similar phenomenon. ${ }^{4}$ The set-up costs of the test are also modest compared with the cost of purchasing a full CPET device, and with a standardised protocol, test results can be compared between centres and potentially within clinical trials. Step climbing testing outside a laboratory setting is potentially hazardous since the patient is not always monitored, and could sustain significant trauma if she/he becomes dizzy or slips. By confining the testing to a laboratory or office setting with a maximum height gain of $17 \mathrm{~cm}$ and continuously monitoring pulse oximetry, we believe that this technique may be safer for patients. Other forms of 'low-technology' exercise testing such as $6 \mathrm{MW}$ require only a long corridor and relatively straightforward training of staff and patients. ${ }^{19}$ However, $6 \mathrm{MW}$ is regarded as a submaximal test of exercise capacity, since the anaerobic threshold is not typically exceeded in during the test. $^{2021}$

As well as demonstrating good agreement between 'gold-standard' parameters of exercise capacity $\left(\mathrm{VO}_{2}\right)$, we also developed and validated novel parameters of exercise performance from the device (3). Previous reports of step climbing have either not recorded or processed detailed data for HR and oxygen saturation during the step test. Clearly it is intuitive and expected that work rate, for example, is correlated closely with $\mathrm{VO}_{2}$. The clinical relevance of this finding is that clinicians are able to objectively quantify exercise capacity without the full CPET device and obtain relevant and valid information.

We demonstrated significant correlation between step climbing desaturation indices and diffusion capacity of the lung (DLCO), replicating the results of a previous 
study. ${ }^{4}$ The best correlations with DLCO were seen with derived measurements of $\mathrm{SpO}_{2}$ such as saturation deviation and desaturation area where the extent of desaturation is averaged or integrated over time, respectively. The simple calculation of absolute desaturation (baseline $\mathrm{SpO}_{2}-$ nadir $\mathrm{SpO}_{2}$ ) was less well correlated with DLCO. The step climbing test therefore also gives the clinician some insight into the gas transfer capacity of the lung, which could be useful in an office setting lacking full lung function tests.

Although there are advantages to a stationary step test as discussed above, there are important differences between this test and a step test performed on a staircase. During normal step climbing, the subject performs work against gravity with each sequential upward step. In our test, most of the antigravity work occurs when lifting the lower foot up onto the step. There is also a step-down phase in the step oximetry test, which is less energy consuming (eccentric muscle contraction) than continuing a sequential upward climb. The subject therefore has regular 'rest' during the test. Energy expenditure and work rate in our step test are calculated only for the ascending work via classical physics equations. These differences in technique probably explain the relatively low work rates reported by our technique, relative to those reported during hospital staircase-based climbing experiments. ${ }^{2}$ We note that despite these limitations, the step test work rate parameter had the best correlation with $\mathrm{VO}_{2}$ (table 3). Another potential limitation is that this study was performed on patients with lung disease, although this should not affect the reliability of results since the study simply checked agreement between the two tests in the same patient. At the present time, we also have no data available to give 'normal ranges' for the various parameters over a large and varied population. Therefore, at this time, the step climbing test and device should be considered a research tool only.

In summary, we provide the first study of laboratory-based, semiautomated and standardised step test using a dedicated device in a cohort of subjects presenting for exercise testing. We believe that step oximetry testing could become a useful functional test in clinical practice.

Contributors BDF conceived the study, built the device, analysed the data and drafted the manuscript. NS collected the data, analysed the data and drafted the manuscript. BV and LF collected the data and revised the manuscript. MRK conceived the study, built the device, analysed the data and revised the manuscript.

Funding The authors have not declared a specific grant for this research from any funding agency in the public, commercial or not-for-profit sectors.

Competing interests None declared.

Patient consent Not required.

Ethics approval All procedures performed in studies involving human participants were in accordance with the ethical standards of the institutional and/or national research committee and with the 1964 Helsinki declaration and its later amendments or comparable ethical standards.

Provenance and peer review Not commissioned; externally peer reviewed.

Data sharing statement Data sharing is possible. Please contact the corresponding author.

Open access This is an open access article distributed in accordance with the Creative Commons Attribution Non Commercial (CC BY-NC 4.0) license, which permits others to distribute, remix, adapt, build upon this work non-commercially, and license their derivative works on different terms, provided the original work is properly cited, appropriate credit is given, any changes made indicated, and the use is non-commercial. See: http://creativecommons.org/licenses/by-nc/4.0/.

\section{REFERENCES}

1. Dal Corso S, de Camargo AA, Izbicki M, et al. A symptom-limited incremental step test determines maximum physiological responses in patients with chronic obstructive pulmonary disease. Respir Med 2013;107:1993-9.

2. Cataneo DC, Cataneo AJ. Accuracy of the stair climbing test using maximal oxygen uptake as the gold standard. J Bras Pneumol 2007:33:128-33.

3. Dal Corso S, Duarte SR, Neder JA, et al. A step test to assess exercise-related oxygen desaturation in interstitial lung disease. Eur Respir J 2007;29:330-6.

4. Fox BD, Langleben D, Hirsch A, et al. Step climbing capacity in patients with pulmonary hypertension. Clin Res Cardiol 2013:102:51-61.

5. Swinburn CR, Wakefield JM, Jones PW. Performance, ventilation, and oxygen consumption in three different types of exercise test in patients with chronic obstructive lung disease. Thorax 1985;40:581-6.

6. Brunelli $\mathrm{A}$, Xiumé $\mathrm{F}$, Refai $\mathrm{M}$, et al. Peak oxygen consumption measured during the stair-climbing test in lung resection candidates. Respiration 2010;80:207-11.

7. Koegelenberg CF, Diacon AH, Irani S, et al. Stair climbing in the functional assessment of lung resection candidates. Respiration 2008;75:374-9

8. Kramer MR, Krivoruk V, Lebzelter J, et al. Quantitative 15 steps exercise oximetry as a marker of disease severity in patients with chronic obstructive pulmonary disease. Isr Med Assoc J 1999;1:165-8.

9. Pollock M, Roa J, Benditt J, et al. Estimation of ventilatory reserve by stair climbing. A study in patients with chronic airflow obstruction. Chest 1993;104:1378-83.

10. Rusanov V, Shitrit D, Fox B, et al. Use of the 15-steps climbing exercise oximetry test in patients with idiopathic pulmonary fibrosis. Respir Med 2008;102:1080-8.

11. Starobin D, Kramer MR, Yarmolovsky A, et al. Assessment of functional capacity in patients with chronic obstructive pulmonary disease: correlation between cardiopulmonary exercise, 6 minute walk and 15 step exercise oximetry test. Isr Med Assoc J 2006;8:460-3.

12. Hansen D, Jacobs N, Bex S, et al. Are fixed-rate step tests medically safe for assessing physical fitness? Eur J Appl Physiol 2011;111:2593-9.

13. Macintyre N, Crapo RO, Viegi G, et al. Standardisation of the singlebreath determination of carbon monoxide uptake in the lung. Eur Respir J 2005;26:720-35.

14. Miller MR, Hankinson J, Brusasco V, et al. Standardisation of spirometry. Eur Respir J 2005;26:319-38.

15. American Thoracic SocietyAmerican College of Chest Physicians. ATS/ACCP Statement on cardiopulmonary exercise testing. Am J Respir Crit Care Med 2003;167:211-77.

16. Bland JM, Altman DG. Statistical methods for assessing agreement between two methods of clinical measurement. Lancet 1986;1:307-10.

17. Bland JM, Altman DG. Transformations, means, and confidence intervals. BMJ 1996;312:1079.

18. Holm S. A simple sequentially rejective multiple test procedure. Scand J Statistics 1979;6:65-70.

19. ATS Committee on Proficiency Standards for Clinical Pulmonary Function Laboratories. ATS statement: guidelines for the six-minute walk test. Am J Respir Crit Care Med 2002;166:111-7.

20. Deboeck G, Niset G, Vachiery JL, et al. Physiological response to the six-minute walk test in pulmonary arterial hypertension. Eur Respir $J$ 2005;26:667-72.

21. Oudiz RJ, Barst RJ, Hansen JE, et al. Cardiopulmonary exercise testing and six-minute walk correlations in pulmonary arterial hypertension. Am J Cardiol 2006;97:123-6. 